\title{
Coupling noise analysis of force and temperature on Taiji-1 interferometer
}

Wang, Juan, Deng, Xiaoqin, Yang, Ran, Luo, Ziren

Juan Wang, Xiaoqin Deng, Ran Yang, Ziren Luo, "Coupling noise analysis of force and temperature on Taiji-1 interferometer," Proc. SPIE 11850, First

Optics Frontier Conference, $118500 U$ (18 June 2021); doi:

$10.1117 / 12.2599590$

SPIE. Event: First Optics Frontier Conference, 2021, Hangzhou, China 


\title{
Coupling noise analysis of force and temperature on Taiji-1 interferometer
}

\author{
Juan Wanga,b, Xiaoqin Deng ${ }^{\mathrm{c}}$, Ran Yang ${ }^{\mathrm{a}}$, and Ziren Luo ${ }^{\mathrm{a}}$ \\ a National Microgravity Laboratory, Institute of Mechanics, Chinese Academy of Sciences \\ (CAS), Beijing 100190, China. \\ ${ }^{\mathrm{b}}$ School of Engineering Science, University of Chinese Academy of Sciences (UCAS), Beijing \\ 100049, China. \\ ${ }^{\mathrm{c}}$ Changchun Institute of Optics, Fine Mechanics and Physics, CAS, Changchun 130033, China.
}

\begin{abstract}
The interference results are associated with the force and temperature fluctuation in Taiji-1. To obtain the maximum effect of these noises, the coupling noise analysis of the force and temperature on the interferometer is discussed. First, the impact mechanism of force and temperature fluctuation is introduced. Then, the mechanical and optical simulation based on a simplified model are performed in turn to get the relationship between the noises and the interference results. The final relationship indicates that coupling effect of the force and temperature fluctuations on the interference is a superposition of the results from the situation with only force or temperature fluctuation, corresponding to the respective frequencies. Based on the relationship, the final amplitudes of interference results caused by the force and temperature fluctuation are $111 \mathrm{pm} @ 0.1 \mathrm{~Hz}$ and $87.47 \mathrm{pm} @ 0.05 \mathrm{~Hz}$, respectively, when the corresponding noises are $50 \mu \mathrm{N} @ 0.1 \mathrm{~Hz}$ and $1.7 \mathrm{mK} @ 0.05 \mathrm{~Hz}$ in the proposed simplified model.
\end{abstract}

Keywords: Taiji-1, noise coupling, interferometry, force noise, temperature noise

\section{INTRODUCTION}

Gravitational waves (GWs) are expected to provide a new window for the astronomy detection. As the GWs detection program in China, Taiji program is designed with a triangle of three spacecraft in orbit of sun. ${ }^{1-3}$ The place noise budget requirement of Taiji program is proposed to reach $5-10 \mathrm{pm} / \sqrt{\mathrm{Hz}}$ with frequencies covering the range of $0.1 \mathrm{mHz}-1 \mathrm{~Hz} .{ }^{1,2}$ As the first verification satellite to Taiji program, Taiji- 1 has been launched to conduct a better verification of the typical technology, such as the high-precision space laser interferometer, the gravitational reference sensor, the micro-Newton thruster and the hyperstatic satellite platform in orbit. ${ }^{4}$ The interferometer plays a great role in the optical layout of the whole satellite because the GWs signal is expected to be extracted through the detecting principle of Michelson interferometer. Hence, it is necessary to satisfy the demanding requirement of the interferometric precision for the purpose of GWs detection. The science objective for the interferometry in Taiji-1 is set to $100 \mathrm{pm} / \sqrt{H z} .{ }^{5}$ The noise analysis, especially from force and temperature field, is the guarantee to fulfill the required accuracy. In the previous work, the effect of the variant force and temperature from micro-Newton thruster and some thermal sources, on interference has been considered separately. ${ }^{4,6}$ However, the whole satellite is affected by external force loading coupling with the temperature field simultaneously, which has been ignored in previous discussion. In this work, we will research the coupling effect of the force and temperature fluctuation on the interference in Taiji-1. Section 2 is devoted to analyze theoretically the influence mechanism of the force and temperature separately. The simulation of the simplified model is conducted to establish the relationship between the coupling noise and interference results in section 3.

Further author information: (Send correspondence to Ran Yang.)

Ran Yang: E-mail: yangran@imech.ac.cn

Juan Wang: E-mail: wangjuan@imech.ac.cn 


\section{INTRODUCTION OF NOISE EFFECTS ON INTERFERENCE}

\subsection{Heterodyne interferometry}

The acousto-optic modulator (AOM) is presented to fulfill the heterodyne interferometry in Taiji-1. The two beams with frequencies of $\omega$ and $\omega_{A O M}$ are brought into interference, and the optical power is detected by the photodetectors: ${ }^{7}$

$$
I=\left|E_{1}+E_{2}\right|^{2}=A^{2}+A^{2} \cos \left[\left(\omega-\omega_{A O M}\right) t+\Delta \varphi_{0}+\Delta \varphi\right],
$$

where $A$ is the amplitude of both signal and reference beams, and $\Delta \varphi_{0}$ is the difference of the initial phase angle. Here, $\Delta \varphi$, the effective phase information, denotes the phase difference when two beams pass through their respective light paths. The stability of the interferometer can be read out from $\Delta \varphi$. Nevertheless, it is susceptible to the external disturbance, such as force and temperature variations. They can result in the so-called optical path noise:

$$
\varphi_{\text {noise }}=k \cdot n \cdot d,
$$

where $k$ is the wave number, $n$ is the refractive index of the optical elements and $d$ is the optical path length. Hence, the measurement phase signal with change of force and temperature in the optical interference elements should be noticed.

\subsection{The impact mechanism of force}

In Taiji-1, if there is no external loading, the satellite is mechanical equilibrium. However, the force from microNewton thrusters is needed for a guarantee of the drag-free control for the test mass. This external load leads to the internal force that tend to back from the initial condition of the elements. ${ }^{8}$ The internal force also concludes those from the process of manufacturing, fabricating and installation before the operation. As a result, the interference elements on the optical bench are deformed.

The change of the optical path length is related to the deformation of the optical elements, which is a function of the force. By using the assumption that the deformation is small, homogeneous and linear, the strain tensor can be given in Eq. (3) in terms of Hooke's law. In Eq. (3), $\sigma_{l l}$ and $\sigma_{i k}$ are stress components, $\sigma$ and $E$ are called Poisson's ratio and Young's modulus, and $\delta_{i k}$ is the Kronecker delta, being 1 when $\mathrm{i}=\mathrm{j}$ and 0 when $\mathrm{i} \neq \mathrm{j} .{ }^{9}$

$$
u_{i k}=\left[(1+\sigma) \sigma_{i k}-\sigma \sigma_{l l} \delta_{i k}\right] / E
$$

The change of refractive index is also related to the mechanical stress according to the photo-elastic effect. It describes a phenomenon that the optical properties of transparent dielectric can be changed from isotropy to anisotropy when it is subjected to the mechanical stress. Hence, the propagation of the light beam in all directions in the anisotropic dielectric is changed and can be described with the index ellipsoid. The general formula of the index ellipsoid is depicted in Eq. (4) when there is no stress subjected to the transparent dielectric.

$$
\frac{x^{2}}{\varepsilon_{x}}+\frac{y^{2}}{\varepsilon_{y}}+\frac{z^{2}}{\varepsilon_{z}}=1
$$

Where $\varepsilon_{x}, \varepsilon_{y}$ and $\varepsilon_{z}$ denote three dielectric constants for an electric field measured along the $\mathrm{x}, \mathrm{y}$ and $\mathrm{z}$ axis, respectively. Also, they are the reflection of the refractive index. However, the shape and dimensions of the index ellipsoid are changed as shown in Eq. (5) when the optical elements are deformed due to the mechanical stress. ${ }^{10}$ The linear coefficients are dependent on dielectric constants, the stress-optical coefficient and the stress component. Hence, the refractive index after the deformation can be derived and hence the change of the optical path difference $(\mathrm{OPD})$ is obtained.

$$
a_{x x} x^{2}+a_{y y} y^{2}+a_{z z} z^{2}+a_{x y} x y+a_{y z} y z+a_{x z} x z=1
$$




\subsection{The impact mechanism of temperature}

The thermal-related phenomenon occurs during the lifetime of Taiji-1, such as heat conduction and thermal radiation. The reason of the absence of the thermal convection is that there is no air flow in the vacuum environment. The energy of the system is changed no matter there is conduction or radiation subjected to the model. It should be noted that the influence of the temperature variations on the OPD is realized also through the thermal stresses, even there are no external forces. It is due to the theory of the linear thermoelastic, which emphasizes the linear addition of the thermal stress and mechanical stress. ${ }^{9}$ Hence, it is necessary to pay attention to the constitutive laws containing not only elastic strain, but also the thermal strain, which leads to the variations of effective optical path length and the refractive index.

To be specific, the energy increase of the system is the subtraction of the absorbed heat and the work done by the internal force. ${ }^{8}$ In literature, the stress formulation of thermo-elasticity has been given in detail. ${ }^{9}$ The thermal stress can be induced to the optical elements because of the energy increase, and then it will lead to the size change of the optical elements:

$$
D L_{l}=\alpha \cdot t \cdot D T,
$$

where $\alpha$ represents the thermal expansion coefficient with a form of $\mathrm{dL} / \mathrm{L} / \mathrm{dT}, t$ is the initial thickness of the optical elements and $D T$ is the temperature instability of the optical elements surfaces. ${ }^{7}$ Here, $D T$ is the temperature result after the thermal radiation. Besides, it is instructive for the selection of the elements that the material with low thermal expansion coefficient can lead to the less local stress between the mental substrate and the optical elements.

On account of the stress birefringence, the influence of the variations of refractive index caused by the temperature actions can be also presented, since the thermal stress has been obtained. Afterwards, the change of OPD caused by the refractive index is:

$$
D L_{n}=\beta \cdot t \cdot D T,
$$

where $\beta$ is the thermal-optic effect with the expression of $\mathrm{dn} / \mathrm{dT}$.

\section{ESTABLISHMENT OF THE NOISE COUPLING RELATIONSHIP}

For Taiji-1 model, the establishment of the actual coupling relationship between noises and interference results is almost impossible due to the sophisticated structure. However, the simulated transfer function to the problem is necessary and available through a series of assumptions in the analysis. For instance, the thermoelastic material in this simulation is assumed to be homogeneous, isotropic and linear, with constant material properties. Besides, the satellite model has been simplified and the influence of the optical path length change is considered as priority.

The whole process of simulation concludes the mechanical and optical simulation. The mechanical simulation gives the directional deformation of all nodes in optical elements. Then the interference results OPD can be read out when laser pass through the optical elements, and this can be realized by the optical ray tracing. The corresponding layout and explanation of the optical path in Taiji-1 can refer to literature. ${ }^{4}$

\subsection{The OPD under the fluctuation of force}

The variant force from micro-Newton thruster is the main force source in Taiji-1. To clarify the maximum effect of thrust, the transient structure analysis is used in this simulation. The application of the force is shown in Fig. 1, where fixed constraint is imposed on the right thrusters, marked in green. It means that four forces are applied with the same value.

The amplitude is set to be $0.1 \mathrm{~N}, 1 \mathrm{~N}$ and $10 \mathrm{~N}$, with a period of $10 \mathrm{~s}$. To get the amplitude-frequency characteristics of the OPD fluctuations, Fast Fourier Transform (FFT) is performed here. ${ }^{4}$ The result fluctuation of OPD varied with a period of 10s. Besides, the relationship between the amplitude of OPD fluctuations and the amplitude of the force shows a linear characteristic. And the corresponding numerical relationship from eight detectors is given respectively in Eq. (8). 

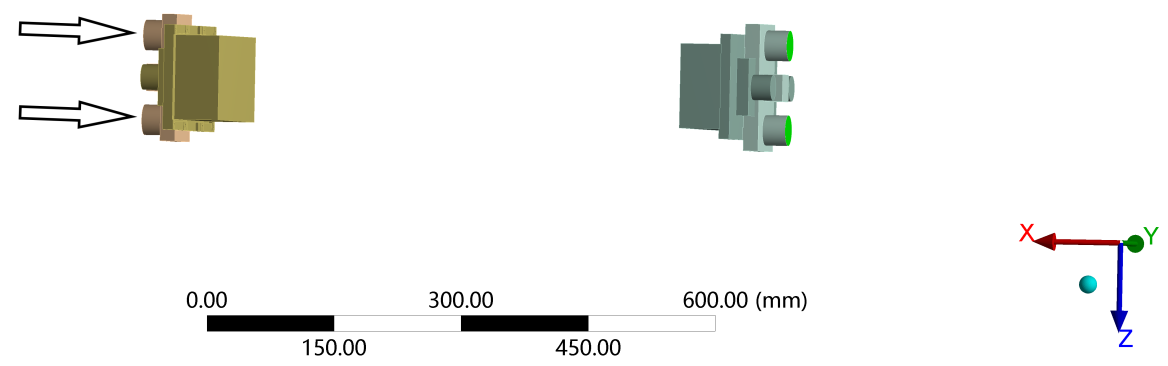

Figure 1. The application of the force.

$$
\begin{aligned}
& D_{1,2}=2.22 \times 10^{-3} \cdot F(\mathrm{~mm}), \\
& D_{3,4}=2.22 \times 10^{-3} \cdot F(\mathrm{~mm}), \\
& D_{5,6}=4.22 \times 10^{-4} \cdot F(\mathrm{~mm}), \\
& D_{7,8}=2.22 \times 10^{-3} \cdot F(\mathrm{~mm}) .
\end{aligned}
$$

\subsection{The OPD under the fluctuation of temperature}

It is necessary to assign the proper heat source since the satellite model is complicated. Thus, the simplified analysis is performed by choosing one of the temperature sources. As shown in Fig. 2, the plate, marked in green, is chosen to play a temperature fluctuation since it is the only one solid connection between the optical bench and the external satellite structure. And the thermal radiation is also considered here by setting the main surfaces, marked in red. The plate is temperature-dependent with the temperature fluctuation of $10^{\circ} \mathrm{C}, 20^{\circ} \mathrm{C}$ and $30^{\circ} \mathrm{C}$, while the ambient temperature is $22^{\circ} \mathrm{C}$. Besides, the temperature fluctuation is set to be different with the force fluctuation with a period of 20 s for the purpose of meeting the actual situation. This thermal activity is analyzed in transient thermal module, with the same fixed constrains shown in Fig. 1, and then the temperature-caused load is imported into transient structure analysis.
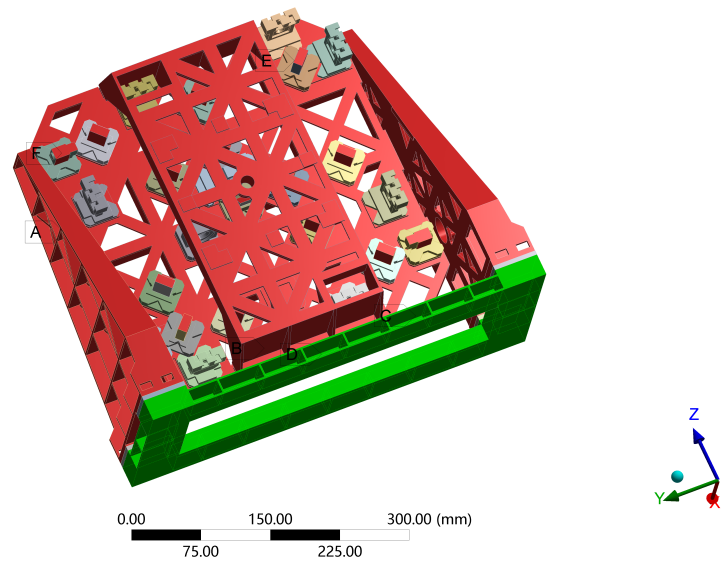

Figure 2. The application of the temperature.

With the same simulation procedure, the result fluctuation of OPD varied with a period of $20 \mathrm{~s} .{ }^{6}$ The corresponding linear relationship between the amplitude of OPD fluctuations and the temperature fluctuation is given in Eq. (9). 


$$
\begin{aligned}
& D_{1,2}=4.718 \times 10^{-6} \cdot \Delta T(\mathrm{~mm}), \\
& D_{3,4}=6.927 \times 10^{-7} \cdot \Delta T(\mathrm{~mm}), \\
& D_{5,6}=5.145 \times 10^{-5} \cdot \Delta T(\mathrm{~mm}), \\
& D_{7,8}=3.037 \times 10^{-6} \cdot \Delta T(\mathrm{~mm}) .
\end{aligned}
$$

\subsection{The OPD under the fluctuations of both force and temperature}

As stated previously, the whole satellite is affected by force loading and the temperature field simultaneously. To investigate the coupling effect of the force and temperature fluctuation on the interference, it is also important to consider the OPD fluctuations in the situation with both force and temperature fields. For example, the sinusoidal force of $0.1 \mathrm{~N}$ with a period of $10 \mathrm{~s}$ is applied on two sets of micro thrusters, and temperature fluctuation amplitude of $10^{\circ} \mathrm{C}$ with a different period of $20 \mathrm{~s}$ is fitted on the plate, simultaneously. Together with transient thermal and transient structure analysis for a duration time of 100s, the deformation of the optical elements can be exported. And the OPD fluctuation can be also obtained with the same optical ray tracing. Consistent with the analysis above, the amplitude-frequency characteristic curve of OPD fluctuations is presented in Fig. 3.

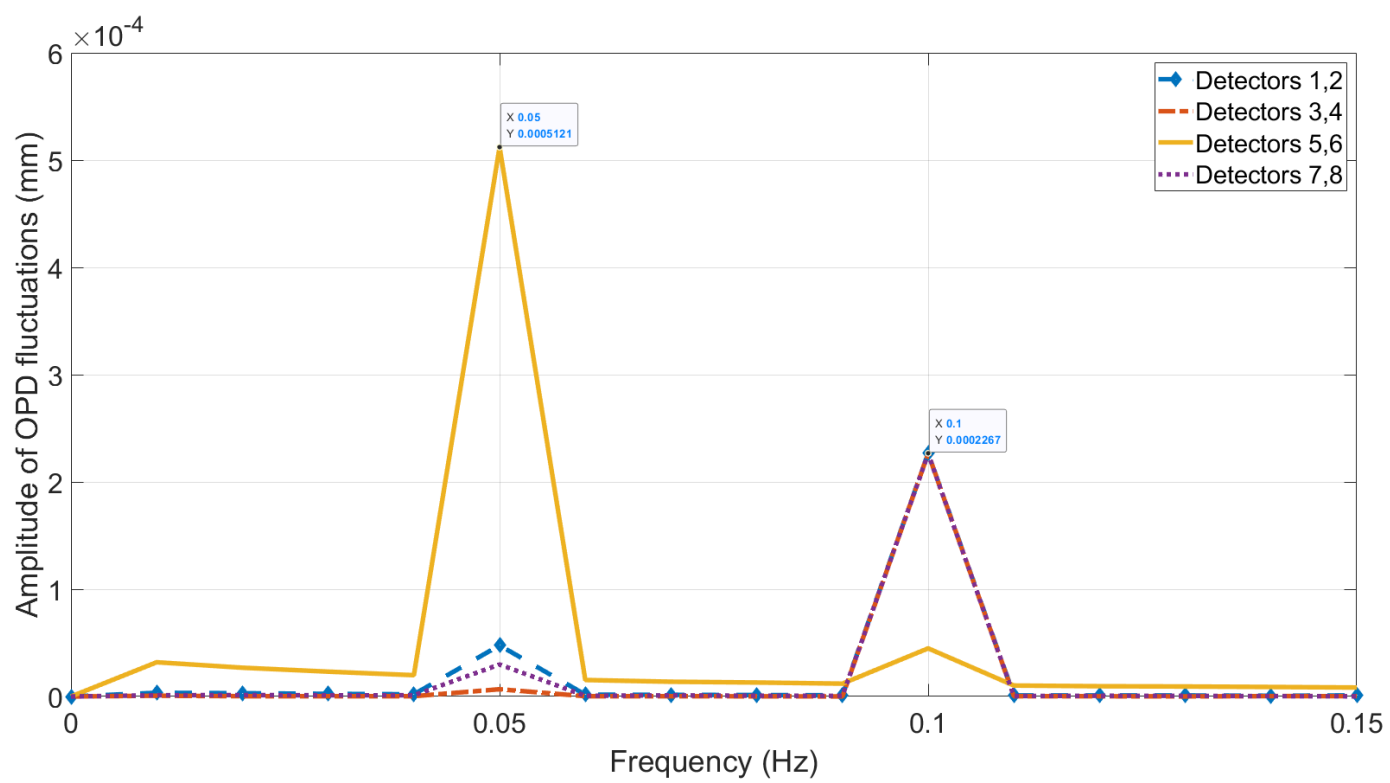

Figure 3. The application of the temperature.

By contrast, the results of $0.1 \mathrm{~N}$ with a period of $10 \mathrm{~s}$ only or $10^{\circ} \mathrm{C}$ with a period of 20 s only can be obtained in Eq. (8) or Eq. (9). The amplitudes of the OPD fluctuations corresponding to three cases are listed in Table 1.

Table 1. The amplitudes of OPD fluctuations in three cases

\begin{tabular}{|l|l|l|l|}
\hline Detectors & $0.1 \mathrm{~N} @ 0.1 \mathrm{~Hz}(\mathrm{~mm})$ & $10^{\circ} \mathrm{C} @ 0.05 \mathrm{~Hz}(\mathrm{~mm})$ & $0.1 \mathrm{~N} @ 0.1 \mathrm{~Hz}+10^{\circ} \mathrm{C} @ 0.05 \mathrm{~Hz}(\mathrm{~mm})$ \\
\hline 1,2 & $2.22 \times 10^{-4} @ 0.1 \mathrm{~Hz}$ & $4.718 \times 10^{-5} @ 0.05 \mathrm{~Hz}$ & $2.262 \times 10^{-4} @ 0.1 \mathrm{~Hz}+4.763 \times 10^{-5} @ 0.05 \mathrm{~Hz}$ \\
\hline 3,4 & $2.22 \times 10^{-4} @ 0.1 \mathrm{~Hz}$ & $6.927 \times 10^{-6} @ 0.05 \mathrm{~Hz}$ & $2.262 \times 10^{-4} @ 0.1 \mathrm{~Hz}+6.756 \times 10^{-6} @ 0.05 \mathrm{~Hz}$ \\
\hline 5,6 & $4.22 \times 10^{-5} @ 0.1 \mathrm{~Hz}$ & $5.145 \times 10^{-4} @ 0.05 \mathrm{~Hz}$ & $4.486 \times 10^{-5} @ 0.1 \mathrm{~Hz}+5.121 \times 10^{-4} @ 0.05 \mathrm{~Hz}$ \\
\hline 7,8 & $2.22 \times 10^{-4} @ 0.1 \mathrm{~Hz}$ & $3.037 \times 10^{-5} @ 0.05 \mathrm{~Hz}$ & $2.262 \times 10^{-4} @ 0.1 \mathrm{~Hz}+2.983 \times 10^{-5} @ 0.05 \mathrm{~Hz}$ \\
\hline
\end{tabular}


We find that the coupling effect of the force and temperature fluctuations on the interference is a superposition of the results from the situation with only force fluctuation and the situation with only temperature fluctuation within a certain error range, corresponding to the respective frequencies.

In Taiji-1, the maxmum force of micro thrusters is $50 \mu \mathrm{N}$, and the temperature control of the scientific instrument in orbit is simulated as $1.7 \mathrm{mK} .{ }^{11}$ As a result, the estimated amplitude of the OPD fluctuations based on the transfer function can be given as:

$$
\begin{aligned}
& D_{1,2}=111 p m @ 0.1 H z+8.02 p m @ 0.05 H z, \\
& D_{3,4}=111 p m @ 0.1 H z+1.18 p m @ 0.05 H z, \\
& D_{5,6}=21.1 p m @ 0.1 H z+87.47 p m @ 0.05 H z, \\
& D_{7,8}=111 p m @ 0.1 H z+5.16 p m @ 0.05 H z .
\end{aligned}
$$

It can be read from Eq. (10) that the maximum effect of the coupling noises in this analysis is $111 \mathrm{pm} @ 0.1 \mathrm{~Hz}$ and 87.47pm@0.05Hz, respectively, when the corresponding noises are $50 \mu \mathrm{N} @ 0.1 \mathrm{~Hz}$ and $1.7 \mathrm{mK} @ 0.05 \mathrm{~Hz}$.

\section{CONCLUSION}

A coupling noise analysis of the force and temperature on Taiji-1 interferometer is depicted in this paper. Based on the impact mechanism description of force and temperature, the simulation to establish the transfer function between external loading and interference result is performed. The results turn out that coupling effect of the force and temperature fluctuation on the interference is a superposition of the results from the situation with only force fluctuation and the situation with only temperature fluctuation, corresponding to the respective frequencies. It should be noted that this result is limited on the specific situation proposed in this paper. However, the simulation results prove the feasibility of the noise coupling analysis of force and temperature using this analysis process. An estimated amplitude of OPD fluctuations caused by the coupling noises is given as $111 \mathrm{pm} @ 0.1 \mathrm{~Hz}$ and $87.47 \mathrm{pm} @ 0.05 \mathrm{~Hz}$, respectively, while the measurement amplitude accuracy of Taiji-1 is required on the magnitude of $100 \mathrm{pm}$. As a result, more accurate model and the experimental validation is still necessary to fulfill the requirement further.

\section{ACKNOWLEDGMENTS}

The authors acknowledge the National Key R\&D Program of China (Grant No. 2020YFC2200104) and the Strategic Priority Research Program of the Chinese Academy of Sciences (Grant No. XDA1502110102) for financial support.

\section{REFERENCES}

[1] Jin., G., "Program in space detection of gravitational wave in chinese academy of sciences," J. Phys.: Conf. Ser. 840 (2017).

[2] Hu., W. and Wu, Y., "The Taiji-1 program in space for gravitational wave physics and the nature of gravity," National Science Review 4, 25-27 (2017).

[3] Liu, H., Luo, Z., and Sha, W., "In-orbit performance of the laser interferometer of Taiji-1 experimental satellite," International Journal of Modern Physics A 36, 2140004 (2021).

[4] Wang, J., Yang, R., and Niu, Y., "Exploration of the coupling between thrust and interference in Taiji-1," International Journal of Modern Physics A (2021).

[5] The Taiji Scientific Collaboration, "China's first step towards probing the expanding universe and the nature of gravity using a space borne gravitational wave antenna," Communications Physics 34, 2140004 (2021).

[6] Deng, X., Yang, R., and Niu, Y., "Preliminary simulation analysis of the temperature fluctuation effect on Taiji-1 laser interferometer," International Journal of Modern Physics A , 2140003 (2021).

[7] LISA Study Team, "LISA technology package architect," (2002).

[8] Landau, L. and Lifshitz, E. M., [Theory of elasticity], Butterworth-Heinemann, U.S.S.R., second ed. (1970). 
[9] Hetnarski, R. and Eslami, M., [Thermal Stresses-Advanced Theory and Applications], Springer Nature Switzerland AG, second ed. (2019).

[10] Born, M. and Wolf, E., [Principles of Optics], seventh(expanded) ed. (1999).

[11] Zhang, X., Liang, H., Tan, H., Feng, J., and Li, H., "Temperature stability of the Taiji-1 satellite in operational orbit," International Journal of Modern Physics A (2021). 